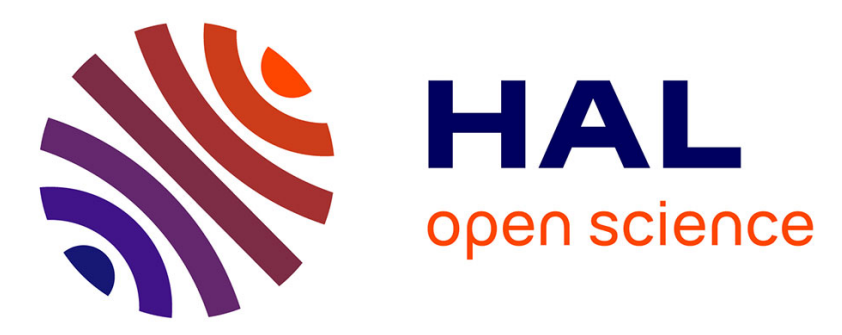

\title{
A simple imaging system to measure velocity and improve the quality of fertilizer spreading in agriculture
}

\author{
Sylvain Villette, Frédéric Cointault, E. Piron, B. Chopinet, M. Paindavoine
}

\section{To cite this version:}

Sylvain Villette, Frédéric Cointault, E. Piron, B. Chopinet, M. Paindavoine. A simple imaging system to measure velocity and improve the quality of fertilizer spreading in agriculture. Journal of Electronic Imaging, 2008, 17 (3), pp.1109-1119. 10.1117/1.2956835 . hal-00448902

\section{HAL Id: hal-00448902 https://hal.science/hal-00448902}

Submitted on 20 Jan 2010

HAL is a multi-disciplinary open access archive for the deposit and dissemination of scientific research documents, whether they are published or not. The documents may come from teaching and research institutions in France or abroad, or from public or private research centers.
L'archive ouverte pluridisciplinaire HAL, est destinée au dépôt et à la diffusion de documents scientifiques de niveau recherche, publiés ou non, émanant des établissements d'enseignement et de recherche français ou étrangers, des laboratoires publics ou privés. 


\title{
A simple imaging system to measure velocity and improve the quality of fertilizer spreading in agriculture
}

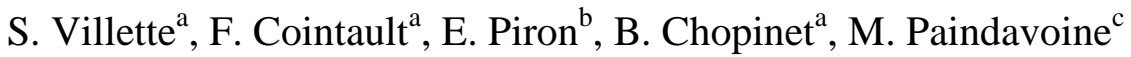 \\ ${ }^{a}$ ENESAD, DSI-LGAP, BP 87999, 21079 Dijon cedex, France \\ s.villette@enesad.fr \\ b Cemagref, Les Palaquins, 03150 Montoldre, France \\ ${ }^{c}$ LE2I, UMR CNRS 5158, Université de Bourgogne, BP 47870, 21078 Dijon cedex, France
}

\begin{abstract}
The management of mineral fertilization using centrifugal spreaders requires the development of spread pattern characterization devices to improve the quality of fertilizer spreading. In order to predict the spread pattern deposition using a ballistic flight model, several parameters need to be determined and especially the velocity of the granules when they leave the spinning disc. This paper demonstrates that a motion blurred image acquired in the vicinity of the disc with a low cost imaging system can provide the three dimensional components of the outlet velocity of the particles. A binary image is first obtained using a recursive linear filter. Then an original method based on the Hough transform is developed to identify the particle trajectories and to measure their horizontal outlet angles, not only in the case of horizontal motion but also in the case of three dimensional motion. The method combines a geometric approach and mechanical knowledge derived from spreading analysis. The outlet velocities are deduced from the outlet angle measurements using kinematic relationships. Experimental results provide first validations for the technique.
\end{abstract}

\section{Introduction}

In agriculture, the nutritive element content of cultivated soils is improved or maintained by regular supplies of mineral fertilizers. In Europe, the application of these granular fertilizers is mainly performed by centrifugal spreaders (Fig. 1) because of their robustness, their simplicity and their low cost.

The mechanical principle of these spreaders consists in feeding a spinning disc with a vertical flow of fertilizer granules. The particles are taken up by a vane, they then undergo a centrifugal acceleration and finally are thrown into the field when they attain the extremity of the vane. The size of the particles is a few millimeters and their outlet velocity can reach more than $40 \mathrm{~m} . \mathrm{s}^{-1}$. In practice, the quality of the spreading depends on many parameters including particle properties and machine characteristics. Consequently, major differences in the quality of the spread pattern deposition are observed in the field because of setting difficulties of the machines. The lack of spreading uniformity has been reported through numerous studies ${ }^{1,2,3}$ when the transverse distribution is assessed in fields. When tests take place in the farmer's field, with his spreader, his fertilizer and his setting, a recent study ${ }^{3}$ in Europe underlines that the quality of the spreading is unsatisfying in $72 \%$ of the tests. The uniformity of the spreading is evaluated by computing the coefficient of variation $(\mathrm{CV})$ of the transverse distribution. Computed with hundred farmers, the mean values of CV were $25 \%{ }^{1}$ or $20.7 \%{ }^{2}$ whereas the spreading quality is considered as satisfying when CV is lower than $15 \%$. The studies ${ }^{1,2,3}$ demonstrates that a first setting adjustment improve significantly the uniformity of the spreading by reducing the CV from approximately $10 \%$ in most cases.

Unfortunately, local over- or under-application involves negative effects not only for the crops $^{1,4}$ but also for the environment ${ }^{5}$, especially in the case of nitrogen fertilizer. 
Consequently, the quality of fertilizer spreading calls for improvements regarding economic and environmental considerations.

To improve the setting of the machines, the usual method uses collection trays placed perpendicularly to the line of travel of the tractor. After the run, the amount of fertilizer collected in each tray is weighed and the coefficient of variation of the transverse distribution is computed. In practice this procedure is tedious for the farmers and consequently tests are rarely carried out to adjust the setting of spreaders.

Moreover, in the context of precision farming, site specific fertilizer management implies the development of rate and uniformity controlled spreaders, using a feedback control system. Consequently, measurement methods and sensors need to be developed to predict the spread pattern deposition of the fertilizer in the field. A prediction based on information obtained in the vicinity of the spinning disc is required to provide a simple spreader test tool for quality diagnoses or an embeddable device to control quality during field work. To achieve these objectives, one method consists in using a ballistic flight model $^{6,7}$ for which the initial conditions of motion need to be measured and especially the velocity of the particles when they leave the spinning disc.

The aim of the present work is to obtain the outlet velocity of the fertilizer particles using a simple and low cost imaging system whatever the shape of the disc (i.e. flat or concave disc).

\section{Various ways to measure fertilizer granule velocities}

Nowadays, Particle Image Velocimetry (PIV) methods are well established measurement techniques to determine particle direction and speed. Nevertheless these techniques do not suit the application. PIV methods ${ }^{8,9}$ employ one or several pulsed sheets of laser light and one or several digital cameras for image recording. These methods are traditionally used to study fluid motions which are made visible by adding small tracer particles. The flow velocity is then deduced from the positions of the tracer particles at two consecutive instances of time. In practice, adaptation of PIV techniques to the measurement of fertilizer granules in the vicinity of a spinning disc is tricky. The main difficulties are linked to the size of the granules (i.e. 2 to $5 \mathrm{~mm}$ ), the no-coplanarity of granule displacements (especially in the case of concave discs), the width of the investigated area around the disc, the motion of specular metallic vanes in the investigated area and the discontinuous nature of the ejected granule flow. The study of the fertilizer outlet velocity by the means of PIV techniques should involve the use of multiple laser sheets and complex image recording systems. Therefore, it appears that these approaches do not meet the objectives of the low cost and robust technique required to preserve a wide field of application for the measurement device. The suitable system has to open up the possibility of designing a simple spreader test tool for static condition uses but also the possibility of extending the technique to an onboard farm implement use.

Various simpler basic methods have already been proposed to estimate the fertilizer outlet velocity in the vicinity of the disc. Hofstee ${ }^{10}$ developed an ultrasonic Doppler device made up of one transmitter and three receivers arranged in a three-dimensional configuration. Grift and Hofstee $^{11}$ developed an optical sensor using two photo sensitive arrays placed perpendicularly with respect to the radius of the disc. The radial component of the outlet velocity was derived from the time difference corresponding to a particle passing each photo sensitive line. As the opening of the measurement cells developed by Hofstee ${ }^{10}$ or Grift and Hofstee ${ }^{11}$ is less than $50 \times 50 \mathrm{~mm}$ these sensors need to be mounted on a rotating arm to turn around the centrifugal disc and to scan the whole angular range of spreading. Cointault and Vangeyte ${ }^{12}$ presented various stroboscopic imaging systems using a single camera to deduce the fertilizer outlet velocity from the displacement of the granules in the image between two successive flashes or lighting strobes. As the real displacement of the granules is directly deduced from 
displacements measured in pixels in the image, the authors implicitly assume that the motion of the granules lies in a plane parallel to the image plane. Consequently the method can not be used in the case of concave disc. Moreover, since the method needs to establish correspondences between granules in the image, it could be difficult to use this method with noisy images.

In contrast to the stroboscopic approach, Villette et al. ${ }^{13}$ demonstrated that velocity information can be extracted from motion blurred images using an optimized Hough transform. Using this technique, the outlet velocity is derived from the outlet angle of the granules when they leave the spinning disc. The most important advantages of this method are the simplicity of image acquisition and the robustness of image processing relative to noisy images. Moreover the method can provide a wide field of view to capture the whole disc vicinity. Nevertheless this previous study was carried out for flat spinning disc and assume the fertilizer trajectories lie in the same horizontal plane in the vicinity of the disc.

The present paper extends the measurement method proposed by Villette et al $^{13}$ to the general case of concave discs which are more common configurations, and demonstrates that the three-dimensional components of the outlet velocity can be extracted from a single motion blurred image.

\section{Design of the imaging system based on motion blurred images}

\subsection{Image acquisition}

For our study, the imaging system was not developed using a commercial spreader, but using a set-up specifically constructed to characterize the trajectories of granules when they leave a spinning disc. The mechanical part of the system is made up of elements similar to those found in a common centrifugal spreader and the imaging system is made up of a single monochrome digital camera $(1008 \times 1018$ pixels $)$. The camera is placed above the granule output flow (Fig. 2) approximately $1 \mathrm{~m}$ above the ejection plane of the disc to help the measurement device retain its compactness. Consequently, an $8.5-\mathrm{mm}$-focal-length is chosen to capture a wide angular range of spreading in the vicinity of the disc. Using this short focal length, the field of view is approximately $1 \times 1 \mathrm{~m}^{2}$ in the disc ejection plane, which is wide enough to cover the wished area. The camera is set vertically so that the view axis is parallel to the rotation axle of the disc. This is of particular interest in the geometric study of image acquisition.

Four traditional spotlights illuminate the granules lengthways during their motion. They are placed $10 \mathrm{~cm}$ above the disc. The light main axis is horizontally directed and the spotlights are oriented so that they illuminate the spreading angular sector.

Depending on the rotational speed of the disc (from 500 to $1000 \mathrm{rpm}$ ), the camera exposure is set from 30 to $60 \mathrm{~ms}$ so that fertilizer particles appear as streaks across the image as shown in figure 3. During this exposure time, the vane rotates approximately $180^{\circ}$ and the streaks due to the motion of the granules are longer than 400 pixels in the interesting area. For example, the exposure time was $35 \mathrm{~ms}$ for the image presented in figure 3 when the rotational speed was $800 \mathrm{rpm}$.

In our work, the tracks due to granule displacements during image integration are considered as a source of information to estimate the velocity of fertilizer particles when they leave the vane. As the outlet velocity of the fertilizer granules cannot be measured directly in the motion blurred image, it will be deduced from the apparent outlet angle $\theta_{\text {out }}$ im of the trajectories with respect to the disc tangential direction (Fig. 3). 
Note that in the case of a flat spinning disc the apparent outlet angle $\theta_{\text {out }}$ im measured in the image is the real horizontal outlet angle.

\subsection{Deducing the three-dimensional components of the outlet velocity}

The physical relationships between the outlet velocity and the horizontal outlet angle $\theta_{\text {out }}$ are provided by the kinematic analysis of the mechanical system. The radial, tangential and vertical components of the velocity vector during the centrifugal acceleration of a fertilizer particle have already been expressed as functions of the velocity of the particle along the vane $^{14}$. In practice, this parameter is unknown, but defining the horizontal outlet angle $\theta_{\text {out }}$ as the angle between the horizontal velocity $v_{R}$ and the tangential velocity $v_{T}$, Villette ${ }^{12}$ demonstrated that each component of the outlet velocity can be expressed as a function of the horizontal outlet angle $\theta_{\text {out }}$ and some other known parameters.

Defining $\theta_{\text {out }}$ as follows:

$$
\theta_{\text {out }}=\arctan \left(\frac{v_{R}}{v_{T}}\right)
$$

the radial component of the velocity is deduced ${ }^{15}$ :

$$
v_{R}=\frac{r_{\text {vane }} \dot{\theta} \tan \theta_{\text {out }}}{1+\tan \theta_{\text {out }} \tan \alpha_{l v}}
$$

where $r_{\text {vane }}$ is the radius of the vane, $\dot{\theta}$ is the rotational speed of the disc and $\alpha_{l v}$ is the horizontal angle of the vane.

Then, the tangential $v_{T}$, the horizontal $v_{H}$ and the vertical $v_{k}$ components are deduced:

$$
\begin{gathered}
v_{T}=\frac{v_{R}}{\tan \theta_{\text {out }}} \\
v_{H}=\frac{v_{R}}{\sin \theta_{\text {out }}} \\
v_{k}=\frac{v_{R}}{\cos \alpha_{l v}} \tan \Omega
\end{gathered}
$$

where $\Omega$ is the angle between the vane and the horizontal plane.

Figure 4 illustrates the configuration parameters of the vane and the location of the velocity components at its extremity. Note that in practice, the geometric parameters of the vane $r_{\text {vane }}$, $\alpha_{l}, \Omega$ and the rotational speed of the disc $\dot{\theta}$ are known parameters.

The kinematic study also demonstrates that the vertical outlet angle $\Omega_{\text {out }}$ can be deduced from the horizontal outlet angle $\theta_{\text {out }}$ and the disc configuration as follows:

$$
\Omega_{\text {out }}=\arctan \left(\frac{\sin \theta_{\text {out }}}{\cos \alpha_{l v}} \tan \Omega\right)
$$

Using the dynamic analysis ${ }^{15}$ of the particle motion on the vane, it is demonstrated that the horizontal outlet angle is a bounded value.

\subsection{Image processing to estimate the horizontal outlet angle}

The aim of the imaging system is to extract the horizontal outlet angles from a motion blurred image in order to deduce the three components of the particle velocities over the entire angular range of spreading. It is worth noting that these angles need to be measured with 
respect to the angular location of the vane which is rotating during the image integration and is not a simple constant orientation in the image. Moreover, in the case of a concave disc, as the trajectories are not parallel to the image plane, the horizontal outlet angles are not the apparent angles measured in the image but will be deduced from them.

\subsubsection{Image preprocessing}

First of all, as we adopted a short focal length (i.e. $8.5 \mathrm{~mm}$ ), the original image is corrected to take into account lens distortion ${ }^{16}$. The second step of image processing consists in obtaining a binary image using a line detection filter. For this study, we used a recursive linear filter inspired by the filter developed by Ziou ${ }^{17}$. To detect line, Ziou models the filter by an approximation of a Gaussian second derivative, whose response impulse is as follows:

$$
f(x)=(a \sin \omega|x|+b \cos \omega|x|) e^{-\alpha|x|}
$$

where $a, b$ are constants and $\alpha, \omega$ are shape parameters.

The author choose the values of the constants $a$ and $b$ so that $\int_{-\infty}^{+\infty} f(x) d x=0$.

This yields $a=-c \alpha$ and $b=c \omega$.

To extend the use of the 1D filter to 2D images, an even regularizing filter is defined. The smoothing function $g(x)$ is chosen so that $g^{\prime \prime}(x)=-f(x)$. The discrete function $f(n)$ and $g(n)$ are then obtained by sampling $f(x)$ and $g(x)$.

Concerning our application, the values of the $a$ and $b$ are computed so that $\sum_{n=-\infty}^{+\infty} f(n)=0$ to obtain a zero response to a constant signal in a discrete space. The sampled impulse responses of the detection filter $f$ and the smoothing function $g$ used for the application are as follows:

$$
\begin{aligned}
& f(n)=(a \sin \omega|n|+b \cos \omega|n|) e^{-\alpha|n|} \\
& g(n)=(A \sin \omega|n|+B \cos \omega|n|) e^{-\alpha|n|}
\end{aligned}
$$

where $a=c\left(e^{-2 \alpha}-1\right), b=2 c e^{-\alpha} \sin \omega, A=-\frac{a\left(\alpha^{2}-\omega^{2}\right)-2 \alpha \omega b}{\left(\alpha^{2}+\omega^{2}\right)^{2}}, B=-\frac{b\left(\alpha^{2}-\omega^{2}\right)+2 \alpha \omega a}{\left(\alpha^{2}+\omega^{2}\right)^{2}}$.

The coefficient $c$ is determined so that $\sum_{-\infty}^{+\infty} g(n)=1$. This yields :

$$
c=\frac{\left(1-2 e^{-\alpha} \cos (\omega)+e^{-2 \alpha}\right)\left(\alpha^{2}+\omega^{2}\right)^{2}}{8 \alpha \omega e^{-2 \alpha} \sin ^{2}(\omega)+2 \alpha \omega\left(e^{-2 \alpha}-1\right)^{2}}
$$

In practice, to process spreading images, we use $\alpha=0.75$ and $\omega=0.01$.

The $\mathrm{Z}$ transforms provides the recursive forms of the derivative filter and the smoothing filter. For each filter, the $\mathrm{Z}$ transforms are broken down into two causal and anticausal parts and the filtering recursion equations are deduced. Expressions of the $\mathrm{Z}$ transforms concerning these filters are given in Villette et $_{\text {al. }}{ }^{13}$.

To apply the line detection on 2D images, the filter is assumed to be separable. Therefore, the image gradient along the columns is obtained by a smoothing operation along rows and a derivative operation along columns. The image gradient along the rows is obtained by a symmetric way. The magnitude image gradient is deduced from these two orthogonal image gradients. A nonmaximum suppression algorithm and a hysteresis thresholding provided a binary image as illustrated in Fig. 5. This step provides various sets of pixels lying on the trajectory axes or resulting from the background of the scene (Fig. 5). 


\subsubsection{Measurement of the horizontal outlet angle}

\subsubsection{Determining apparent outlet angles in the image}

The third step of image processing consists in recognizing the lines in the binary image. This step is carried out by the use of the polar Hough transform ${ }^{18,19}$ which is of particular interest in the study of centrifugal spreading ${ }^{13}$.

Actually, using the angle-radius description of a line $\left(\theta_{T}, \rho_{T}\right)$ and placing the origin of the coordinate system at the center of the spinning disc (Fig. 6), the apparent outlet angle of one trajectory is simply deduced from the radius parameter $\rho_{T}$ of the identified line:

$$
\theta_{\text {out_im }}=\arccos \left(\frac{\rho_{T}}{r_{\text {vane_pix }}}\right)
$$

where $r_{\text {vane_pix }}$ is the radius of the vane expressed in pixels in the image.

In practice, the meter per pixel conversion factor is determined in the ejection plane (i.e. the horizontal plane defined by the ejection point at the extremity of the vane) to derive $r_{\text {vane_pix }}$ from the real radius of the vane measured on the set-up.

Therefore, computing the polar Hough transform with respect to the center of the spinning disc, the apparent outlet angles $\theta_{\text {out } i m}$ are deduced from the location $\left(\theta_{T}, \rho_{T}\right)$ of the peaks in the Hough space. In this way, we directly obtain the angle between the trajectory image and the disc tangential direction, whatever the vane location.

The corresponding angular location of the vane is also simply deduced:

$$
\theta_{\text {vane }}=\theta_{T}+\theta_{\text {out_im }}
$$

The choice of the origin of the coordinate system at the disc center to compute the Hough transform provides several advantages. Taking into account that the horizontal outlet angle is a bounded value, the lower and the upper bounds of $\rho_{T}$ are computed as functions of the disc configuration. Moreover the investigated zone in the image is chosen as a ring centered on the centre of the spinning disc. In practice the ring is approximately 300 pixel wide.

These choices have useful consequences ${ }^{13}$ :

- the size of the accumulator array for the Hough space is reduced,

- the number of relevant pixels considered in the image is reduced,

- the Hough space is quantized optimally avoiding peak spreading or peak extension ${ }^{20,21,22}$,

- peak searching in the Hough space is more efficient and the robustness of the trajectory identification is improved,

- lines whose orientations are improbable are naturally rejected and are not identified as trajectories.

In the Hough space, peak extraction is carried out iteratively, identifying the global maximum, deducing the line parameters and subtracting from the Hough space the Hough transform of the corresponding segment. The values of the line parameters are improved when a forth processing step is carried out. This processing consists in using the peak location in the Hough space to design a thin region of interest in the binary image so that this region covers the set of pixels voting for the considered peak. The line parameters are then accurately deduced from the inertia axis of the pixels lying in this thin window. This processing method has been used in this work to avoid discretization regarding final velocity estimations owing to the Hough space use and to prove that motion blurred images are accurate source of information. 
Figure 7 presents an example of trajectory identification on a detail of an image. It is worth noticing that improbable segments (with respect to the main flow) are not identified as trajectories.

In the case of a flat disc, assuming that the trajectories are in a horizontal plane parallel to the image plane, the angle $\theta_{\text {out }} i m$ deduced from the Hough transform is the horizontal outlet angle $\theta_{\text {out }}$ and can be directly used to compute the outlet velocity. Nevertheless, in the case of a concave disc, the trajectories are not in the same plane during spreading and are not parallel to the image plane. Consequently, there is a difference between the orientation of the trajectories in the image and the orientation that their horizontal components actually have. This angular bias is illustrated in figures 8 and 9 .

\subsubsection{Deducing actual horizontal outlet angles}

The expression of the angular bias $\varepsilon_{\theta}$ is established using a geometric decomposition of the image acquisition based on the pinhole camera model and assuming that the rotation axle of the disc is parallel to the view axis of the camera. In this paper, the rotation axle is assumed to be vertical to simplify explanations. A previous calibration stage provided the required intrinsic camera parameters (e.g. pixel size, focal length and principal point).

The method consists in noting that the distance between the lens focus and the trajectory is unknown except for the ejection point. This point belongs to the trajectory but it also corresponds to the extremity of the vane that define the horizontal ejection plane $\Pi$ and its altitude is perfectly known in the three-dimensional scene.

Considering one granule leaving the extremity of the vane of a concave disc, several items are defined to decompose the geometric arrangement of the scene and the image acquisition (Fig. 10), as follows:

- E is the ejection point of the granule,

- T is the trajectory of the granule (the vertical angle of $\mathrm{T}$ with respect to $\Pi$ is $\Omega_{\text {out }}$ ),

- $\mathrm{T}_{\text {Hor }}$ is the horizontal projection of $\mathrm{T}$ in $\Pi$,

- $\mathrm{M}$ is a point of the trajectory $\mathrm{T}$,

- $\mathrm{E}_{\mathrm{i}}, \mathrm{M}_{\mathrm{i}}, \mathrm{T}_{\mathrm{im}}$ and $\mathrm{T}_{\mathrm{Hor} \_\mathrm{im}}$.are the image of $\mathrm{E}, \mathrm{M}, \mathrm{T}$ and $\mathrm{T}_{\mathrm{Hor}}$ respectively in the CCD plane,

- $\mathrm{C}$ is the focus point,

- $\mathrm{P}$ is the principal point in the image.

Four right-handed Cartesian coordinate systems are also defined (Fig. 10), as follows:

- (E, $\left.\boldsymbol{i}_{\boldsymbol{E} T \boldsymbol{H}}, \boldsymbol{j}_{\boldsymbol{E} T \boldsymbol{H}}, \boldsymbol{k}_{\boldsymbol{E} \boldsymbol{T H}}\right)$ is so that $\boldsymbol{i}_{\boldsymbol{E} T \boldsymbol{H}}$ is along $\mathrm{T}_{\mathrm{Hor}}$ in the horizontal ejection plane, following the motion direction of the granule and $\boldsymbol{k}_{\boldsymbol{E} T \boldsymbol{H}}$ is an upward vertical vector. In this frame, the coordinates of one point $\mathrm{M}$ are $\left(x_{E T H}(\mathrm{M}), y_{E T H}(\mathrm{M}), z_{E T H}(\mathrm{M})\right)$ and are expressed in meter.

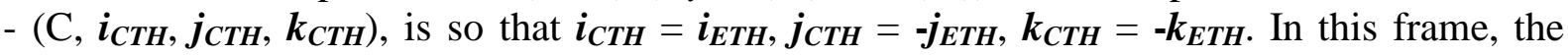
coordinates of one point $\mathrm{M}$ are $\left(x_{C T H}(\mathrm{M}), y_{C T H}(\mathrm{M}), z_{C T H}(\mathrm{M})\right)$ and are expressed in meter.

- (Ei, $\left.\boldsymbol{i}_{\boldsymbol{E} T \boldsymbol{i}}, \boldsymbol{j}_{\boldsymbol{E} T \boldsymbol{H} \boldsymbol{i}}\right)$ is so that $\boldsymbol{i}_{\boldsymbol{E} T \boldsymbol{i} \boldsymbol{i}}$ is along $\mathrm{T}_{\text {Hor_im }}$ and $\boldsymbol{j}_{\boldsymbol{E} T \boldsymbol{H} \boldsymbol{i}}$ has the same direction than $\boldsymbol{j}_{\boldsymbol{E} T \boldsymbol{H}}$. $\boldsymbol{i}_{\boldsymbol{E} T \boldsymbol{i} \boldsymbol{i}}$ and $\boldsymbol{j}_{\boldsymbol{E} T \boldsymbol{H} \boldsymbol{i}}$ belong to the image plane. In this frame, the coordinates of one point $\mathrm{M}_{\mathrm{i}}$ are $\left(x_{E T H i}\left(\mathrm{M}_{\mathrm{i}}\right), y_{E T H i}\left(\mathrm{M}_{\mathrm{i}}\right), z_{E T H i}\left(\mathrm{M}_{\mathrm{i}}\right)\right)$ and are expressed in pixel.

- $\left(E i, \boldsymbol{i}_{E T i}, \boldsymbol{j}_{E T i}\right)$ is so that $\boldsymbol{i}_{\boldsymbol{E} T \boldsymbol{i}}$ is along $\mathrm{T}_{\mathrm{im}}$ following the motion direction of the granule. $\boldsymbol{i}_{\boldsymbol{E} T \boldsymbol{H} \boldsymbol{i}}$ and $\boldsymbol{j}_{\boldsymbol{E} T H i}$ belong to the image plane. In this frame, the coordinates of one point $\mathrm{M}_{\mathrm{i}}$ are $\left(x_{E T i}\left(\mathrm{M}_{\mathrm{i}}\right), y_{E T i}\left(\mathrm{M}_{\mathrm{i}}\right), z_{E T i}\left(\mathrm{M}_{\mathrm{i}}\right)\right)$ and are expressed in pixel.

Since $\mathrm{M}$ belongs to the trajectory $\mathrm{T}$, the coordinates of $\mathrm{M}$ in $\left(\mathrm{C}, \boldsymbol{i}_{\boldsymbol{C T H}}, \boldsymbol{j}_{\boldsymbol{C} T \boldsymbol{H}}, \boldsymbol{k}_{\boldsymbol{C T H} \boldsymbol{H}}\right)$ are: 


$$
\left(\begin{array}{l}
x_{C T H}(\mathrm{M}) \\
y_{C T H}(\mathrm{M}) \\
z_{C T H}(\mathrm{M})=z_{C T H}(\mathrm{E})-x_{E T H}(\mathrm{M}) \times \tan \Omega_{\text {out }}
\end{array}\right)
$$

As the pixels of the CCD are square, considering the pinhole camera model, the coordinates of $\mathrm{M}_{\mathrm{i}}$ in $\left(\mathrm{Ei}, \boldsymbol{i}_{E T H i}, \boldsymbol{j}_{E T H i}\right)$ are:

$$
\left\{\begin{array}{c}
x_{E T H i}\left(\mathrm{M}_{\mathrm{i}}\right)=\frac{f}{\Delta p} \frac{x_{C T H}(\mathrm{M})}{z_{C T H}(\mathrm{M})}+x_{E T H i}(\mathrm{P}) \\
y_{E T H i}\left(\mathrm{M}_{\mathrm{i}}\right)=\frac{f}{\Delta p} \frac{-y_{C T H}(\mathrm{M})}{z_{C T H}(\mathrm{M})}+y_{E T H i}(\mathrm{P})
\end{array}\right.
$$

where $\Delta p$ is the width of pixels, $f$ is the focal length and $\left(x_{E T H i}(\mathrm{P}), y_{E T H i}(\mathrm{P})\right)$ are the coordinates of the principal point $\mathrm{P}$ expressed in $\left(\mathrm{Ei}, \boldsymbol{i}_{\boldsymbol{E} T \boldsymbol{i}}, \boldsymbol{j}_{\boldsymbol{E} T \boldsymbol{H}}\right)$.

Then the angular bias $\varepsilon_{\theta}=\theta_{\text {out }}-\theta_{\text {out_im }}$ between $\mathrm{T}_{\mathrm{im}}$ and $\mathrm{T}_{\mathrm{Hor} \_\mathrm{im}}$, is so that:

$$
\tan \varepsilon_{\theta}=\frac{y_{E T H i}\left(\mathrm{M}_{\mathrm{i}}\right)}{x_{E T H i}\left(\mathrm{M}_{\mathrm{i}}\right)}
$$

Substituting (13) in (14) and operating the geometrical decomposition described in appendix, the relationships becomes:

$$
\tan \varepsilon_{\theta}=\frac{-y_{\text {ETHi }}(P) \tan \Omega_{\text {out }}}{\frac{f}{\Delta p}-x_{\text {ETHi }}(\mathrm{P}) \tan \Omega_{\text {out }}}
$$

The coordinates of $\mathrm{P}$ established in $\left(\mathrm{Ei}, \boldsymbol{i}_{\boldsymbol{E} T \boldsymbol{H} \boldsymbol{i}}, \boldsymbol{j}_{\boldsymbol{E} T \boldsymbol{H} \boldsymbol{i}}\right)$ are expressed as functions of the coordinates in $\left(\mathrm{Ei}, \boldsymbol{i}_{E T i}, \boldsymbol{j}_{E T i}\right)$ as follows:

$$
\left\{\begin{array}{l}
x_{E T H i}(\mathrm{P})=x_{E T i}(\mathrm{P}) \cos \left(\varepsilon_{\theta}\right)-y_{E T i}(\mathrm{P}) \sin \left(\varepsilon_{\theta}\right) \\
y_{E T H i}(\mathrm{P})=x_{E T i}(\mathrm{P}) \sin \left(\varepsilon_{\theta}\right)+y_{E T i}(\mathrm{P}) \cos \left(\varepsilon_{\theta}\right)
\end{array}\right.
$$

As the location of $\mathrm{T}_{\text {Hor_im }}$ cannot be obtained directly from the image of the scene, $x_{E T H i}(\mathrm{P})$ and $y_{E T H i}(\mathrm{P})$ are replaced in (15) using (16). Then, after mathematical simplifications the expression of the angular bias $\varepsilon_{\theta}$ is established as follows:

$$
\varepsilon_{\theta}=\arcsin \left(-y_{E T i}(\mathrm{P}) \tan \left(\Omega_{\text {out }}\right) \frac{\Delta p}{f}\right)
$$

Substituting $\varepsilon_{\theta}$ with $\left(\theta_{\text {out }}-\theta_{\text {out_im }}\right)$ and substituting $\Omega_{\text {out }}$ with Eq. (6), the expression of the correct horizontal outlet angle is established as follows after some mathematical transformations:

$$
\theta_{\text {out }}=\arctan \left(\frac{\sin \theta_{\text {out_im }}}{\cos \theta_{\text {out_im }}+y_{E T i}(P) \frac{\Delta p}{f} \frac{\tan \Omega}{\cos \alpha_{l v}}}\right)
$$

Using this approach and a single motion blurred image, the horizontal outlet angles are correctly measured for the whole angular range of spreading although the trajectories are not 
parallel to the image plane. Consequently, the components of the velocity (Eqs. (2) to (5)) can be deduced from the kinematic analysis of the motion.

\section{Experimental results}

The imaging system was first assessed by means of a static model simulating a trajectory with a physical line (i.e. tightened rope) for two vertical angles and various locations of the simulated vane. The maximum difference obtained between the reference horizontal outlet angle and the value deduced from the imaging system was $0.23^{\circ}$, which is very satisfying accuracy for our application.

In the case of a flat spinning disc, velocity measurements based on motion blurred images were compared to stroboscopic measurements. Plastic spheres of $6 \mathrm{~mm}$ diameter were used for this test to assume the same drag coefficient for every particle. For approximately 60 test trajectories, the mean velocity $\left(43.7 \mathrm{~m} . \mathrm{s}^{-1}\right)$ and the standard deviation $\left(0.6 \mathrm{~m} . \mathrm{s}^{-1}\right)$ obtained by both methods were the same. Comparing the estimations of the velocity for each trajectory, the standard deviation of the estimation differences was $0.6 \mathrm{~m} . \mathrm{s}^{-1}$, that is to say $1.3 \%$ of the global mean velocity.

In the case of a concave disc, the mean of the vertical outlet angle estimated by image processing was compared to estimations deduced from the location of granule impacts on a screen placed in the vicinity of the disc. The location of the impacts were recorded by using protected sheet of paper covered by a carbon film. The mean vertical height of the impacts was measured with respect to the outlet altitude of the fertilizer particles when they leave the vane. To compare the mean value of the vertical outlet angle deduced from images with the mean value deduced from impacts, the theoretical ballistic flight of the particle was computed. For various spreading situations, the maximum difference between the mean values deduced from the image processing and the reference values was $0 \cdot 26^{\circ}$.

The velocity components were then measured on fertilizer spreading images. Figure 11 presents an example of results obtained for potassium chloride and using a concave disc $(\Omega=$ $\left.10 \cdot 7^{\circ}\right)$ equipped with pitched vanes $\left(r_{\text {vane }}=391 \mathrm{~mm}, r_{p}=-24 \mathrm{~mm}\right)$, rotating at $800 \mathrm{rpm}$.

Until recently, tests and trials were carried out in indoor controlled laboratory conditions for various fertilizers (potassium chloride, ammonium nitrate and compound fertilizer), various lighting conditions (only spotlights, spotlights and indoor daylight illumination, spotlights and ambient electrical laboratory lighting) and various background situations (plastic or canvas tarpaulin, light land). Figures $12 \& 13$ illustrate the results obtained for the same fertilizer spreading configuration but for two different lighting and background conditions involving low quality images. Concerning these figures, the test was carried out with potassium chloride and the scene was illuminated by four spotlights and the ambient electrical lighting of the laboratory. When creased canvas tarpaulin is used for the background (Fig. 12), the mean value of the outlet velocity computed with seven images is $35.28 \mathrm{~m} . \mathrm{s}^{-1}$ and the standard deviation is $0.72 \mathrm{~m} . \mathrm{s}^{-1}$. When light land with white particles (Fig. 13) is used, the outlet velocity computed with seven images is $35.23 \mathrm{~m} . \mathrm{s}^{-1}$ and the standard deviation is $0.75 \mathrm{~m} . \mathrm{s}^{-1}$. In spite of the differences in the image acquisition conditions, the mean outlet velocities deduced from the image analysis are not significantly different. As the fertilizer and the mechanical device were the same in both situations it is natural for fertilizer outlet velocity to be the same. These results underline the robustness of the imaging system. 
Nevertheless, one aspect of our future work will consist in assessing the imaging system under in-field conditions especially concerning lighting conditions and adapting the device to real working conditions. To reduce alterations due to outdoor lighting, several ways are conceivable. Improved spotlights with reflector or lens can be used to concentrate illumination near to the ejection plane of the granules. The use of specific radiation such as infrared illumination can also be used to enhance the granule trajectories in the image. The use of a protection box is also a solution to reduce the influence of outdoor lighting on the image acquisition on a 30-centimeter-width-ring around the disc.

When the quality of the grey level image deteriorates, the binary filtered image shows more and more line discontinuities and points without relationship with the trajectories (Fig. 12 \& 13). Nevertheless, the line detection step limits the sensitivity to luminosity variations due to fertilizer dust suspensions, lighting or background heterogeneity. Moreover, the robustness of the adapted Hough transform provides a satisfying trajectory identification in the case of noisy binary images limiting false-positive detections. For our application, it is worth noticing that false-negative detections are not problematic as long as trajectories are identified over the whole angular range of spreading.

For this work, the processing algorithms were developed with Matlab v6.1 (The MatWorks, Inc. Natick, USA) and were run with an Intel Pentium $43 \mathrm{Ghz}$ Processor. Considering spreading images such as the one presented in figure 3 , the processing time was $3.5 \mathrm{~s}$ for applying the line detection filter and obtaining the binary image of the rectangular window $(1008 \times 500$ pixels $)$ which covers the relevant investigated ring in the disc vicinity. Computing the Hough transform takes $4 \mathrm{~s}$ and the determination of line parameters using inertia axes of sets of pixels can take $25 \mathrm{~s}$. So the total computation time is more than $30 \mathrm{~s}$. It is worth noting that, these algorithms have been developed to prove that simple blurred motion images are accurate source of information to estimate fertilizer velocity when granules are projected by concave discs. The imaging system and the processing algorithms have now to be improved regarding the computation time and the application objectives.

Concerning the design of farm mobile test tools to characterize the spread pattern without using collection trays and running the tractor in the field,. Regarding the design of an onboard sensor to control the spreading, a half-minute-computation time would present a first interest compared to the current lack of information. This should be especially interesting for a scenario where experimental models would be used to set the spreader by taking into experimental setting laws and regular fertilizer behavior measurements deduced from the sensor. Nevertheless, faster processing algorithms providing information concerning spreading features each $2 \mathrm{~s}$ for example, would obviously better suit the objective of a direct feedback control system.

The reduction of the computation time will be obtained by reducing the image resolution and the algorithm complexity according to the practical accuracy required by the application. For example, deducing line parameters directly from the Hough space without any post processing (i.e. inertia axis determination) takes $6 \mathrm{~s}$ instead of $25 \mathrm{~s}$, whereas the difference regarding the estimation of the velocity mean value is lower than $1 \%$. The reduction of the computation time will also be obtained by choosing more efficient programming language and computing systems.

\section{Conclusion}

This study demonstrates that the velocity of fertilizer particles ejected by a centrifugal spreader can be measured by means of motion blurred images provided by a low cost imaging 
system. Combining the kinematic study of the fertilizer motion on the vane and the geometric analysis of image acquisition, the 3D components of the outlet velocity can be deduced from motion blurred images in the case of a traditional concave disc.

The estimation of outlet velocities is useful in predicting spread patterns using ballistic flight models. This opens up the possibility of implementing simple spreader test tools for quality diagnoses and further on-board sensors for in-field spreading control.

Further studies will consist in using this imaging system to estimate the angular distribution of the fertilizer flow in the vicinity of the spinning disc; assessing the imaging system in outdoor uncontrolled conditions and reducing the computation time according to the practical accuracy required by the focused applications.

\section{References}

1. S. Tissot, G. Queron and O. Miserque, "Tolérance d'une culture de froment à l'égard de l'hétérogénéité d'épandage des engrais azotés, [Tolerance of wheat crop towards the spreading heterogeneity of nitrogen manure,]" Biotechnology Agronomy Society Environment 3(3), 247-252, (1999).

2. P.-Y. Leterme, "Précision des épandages : une situation contrastée, [Spreading accuracy: a contrasted situation,]" Perspectives Agricoles 263, 62-64 (2000)

3. D. Roussel, "Spreader field tests: The Dynatest method and spreading practices observations," in Proc. $2^{\text {nd }}$ International Symposium on centrifugal fertilizer spreading, Montoldre, France, pp. Q1-Q5, (2006).

4. H. T. Sogaard and P. Kierkegaard, "Yield reduction resulting from uneven fertilizer distribution." Transactions of ASAE 36(6), 1749-1752, (1994).

5. S. Tissot, O. Miserque, O. Mosrade, B. Huyghebaert, and J. P. Destain, "Uniformity of Nfertilizer spreading and risk of ground water contamination," Irrigation and drainage 51(1), 17-24, (2002).

6. R. M. Mennel, A. R. Reece, "The theory of the centrifugal distributor, III: Particle trajectories," Journal of Agricultural Engineering Research 8(1), 78-84, (1963).

7. R.E. Pitt, G. S. Farmer, L. P. Walker, "Approximating equations for rotary distributor spread patterns," Transactions of the American Society of Agricultural Engineers 25(6), 1544-1552, (1982).

8. J Westerweel, "Fundamentals of digital particle image velocimetry," Measurement and Science and Technology, 8, 1379-1392, (1997).

9. M. Stanislas, J.-C. Monnier, "Practical aspects of image recording in particle image velocimetry," Measurement Science and Technology, 8, 1417-1426, (1997).

10. J. W. Hofstee, "Handling and spreading of fertilizers : Part 3, Measurement of particle velocities and directions with ultrasonic transducers, theory, measurement system and experimental arrangements," Journal of Agricultural Engineering Research 58, 1-16, (1994).

11. T. E Grift., J. W.Hofstee, "Measurement of velocity and diameter of individual fertilizer particles by an optical method," Journal of Agricultural Engineering Research 66, 235238, (1997). 
12. F. Cointault, J. Vangeyte, "Photographic imaging systems to measure fertilizer granule velocity during spreading," in Proc. The International Fertilizer Society, proceeding 555, 28 pages, $(2005)$.

13. S. Villette, F. Cointault, B. Chopinet, M. Paindavoine, "Optimizing Hough transform for fertilizer spreading optical control," Optical Engineering 45(2), Article n ${ }^{\circ}$ 027006, 11 pages, (2006).

14. S. Villette, F. Cointault, E. Piron, B. Chopinet, "Centrifugal spreading : an analytical model for the motion of fertilizer particles on a spinning disc," Biosystems Engineering 92(2), 157-164, 2005.

15. S. Villette, "Estimation de vitesse par analyse d'images acquises en filé : Application à la caractérisation de la distribution centrifuge de granules d'engrais, [Velocity estimation using motion blurred images: Application to the characterization of centrifugal distribution of granular fertilizer,]" PhD Thesis, Université de Bourgogne, France (2006).

16. J. Heikkilä and O. Silvén, "A four-step camera calibration procedure with implicit image correction," in Proc. IEEE Computer Society Conference on Computer Vision and Pattern Recognition, San Juan, Puerto Rico, 1106-1112, (1997).

17. D. Ziou, "Line detection using an optimal IIR filter," Pattern Recognition 24(6), 465-478, (1991).

18. P. V. C. Hough, "Methods and means for recognizing complex patterns," US patent $\mathrm{n}^{\circ} 3$ 069 654, 18 December 1962.

19. R. O. Duda, P. E. Hart, "Use of Hough transformation to detect lines and curves in pictures," Communications of the Association for Computing Machinery, 15(1), 11-15, (1972).

20. W. C. Y. Lam, L. T. S. Lam, K. S. Y. Yuen, D. N. K. Leung, "An error analysis on quantizing the Hough space," in Proc. ICSP'93, 946-949, (1993).

21. Hansen K, Andersen J D, 1997. "Understanding the Hough transform: Hough cell support and its utilisation," Image and Vision Computing 15, 205-218, (1997).

22. S. Y. Yuen, C. H. Ma, "An investigation of the nature of parameterization for the Hough transform," Pattern Recognition, 30(6), 1009-1040, (1997).

\section{Biographies}

Sylvain Villette received his engineering degree in agronomic sciences from ENITA-Dijon, France, in 1994. He obtained his $\mathrm{PhD}$ in signal and image processing from Burgundy University, France, in 2006. He has been teaching in the ENESAD (agronomical engineering school and institute of Dijon, France) since 2001 and he is currently an assistant professor of agricultural machinery engineering. His research work deals with farm implement design and control, especially for mineral fertiliser spreading.

Frédéric Cointault gets his $\mathrm{PhD}$ in Intrumentationnand Image Computing at the University of Burgundy in 2001 and is currently an associate professor at ENESAD (Higher Educational Institute of Agronomy of Dijon, France). His research deal with the use of image processing techniques for characterization of centrifugal spreading process (high speed imagery and motion estimation) and for the characterization of wheat crop by color, spectral and texture analysis (counting, diseases).

Bernard Chopinet obtained his $\mathrm{PhD}$ in electronic engineering from the University of Burgundy (Dijon), France, in 1973. Since 2000, he has been in charge of the Laboratory of Agro-engineering and Processes (GAP) of ENESAD (The National School of Higher 
Agronomical Studies) and from 2004 to 2006, he was Head of the Cemagref/ENESAD joint research unit on Sensors and Processes for Precision Farming (UMR CPAP). His main domain of research concerns the control of farming inputs. He was also member of the National Committee for the Evaluation of Researchers of the French Ministry of Agriculture (CNECA) from 1998 to 2004. 


\section{List of figure captions}

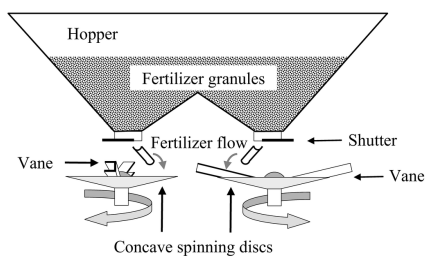

Figure 1: Centrifugal spreader principle using two spinning discs.

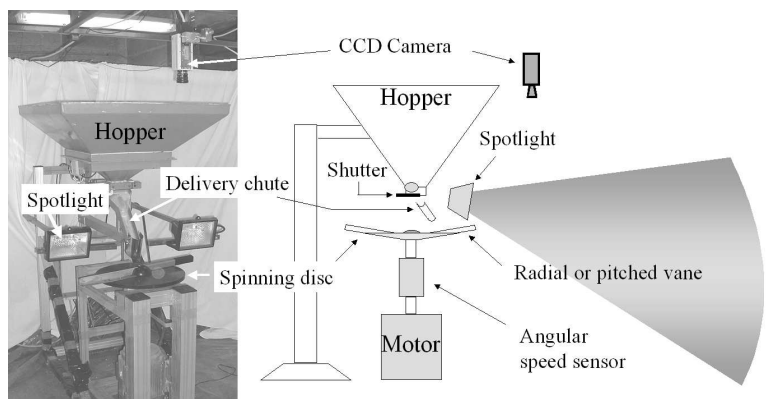

Figure 2: Mechanical and imaging set-up.

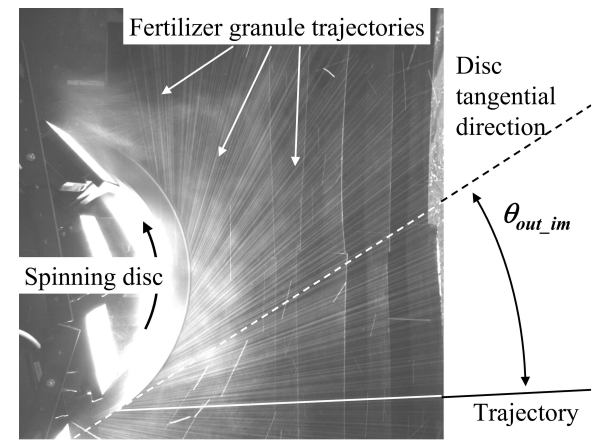

Figure 3: Example of motion blurred image. $\theta_{\text {out_im }}$ is the apparent outlet angle.

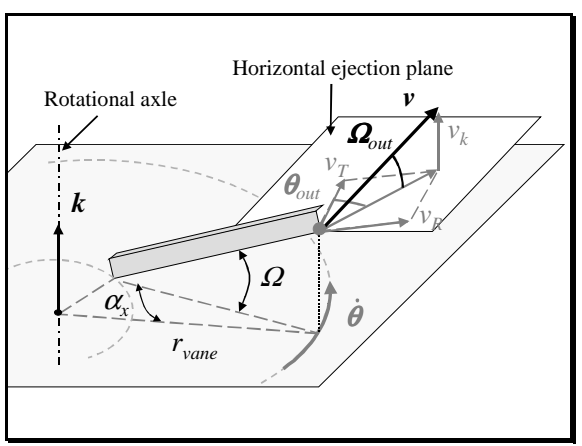

Figure 4 : Illustration of the vane configuration parameters and the velocity components of the granule when it leaves the vane. $\boldsymbol{k}$ is a vertical vector along the rotational axle of the disc; rvane is the radius of the vane; $\alpha \mathrm{x}$ and $\Omega$ are respectively the horizontal pitch angle and the vertical angle of the vane; $\dot{\theta}$ is the rotational speed of the vane; $\theta_{\text {out }}$ and $\Omega_{\text {out }}$ are respectively the horizontal and the vertical outlet angles of the granule; $v$ is outlet velocity vector; $v_{T}, v_{R}$ and $v_{K}$ are respectively the tangential, radial and vertical components of the velocity. 

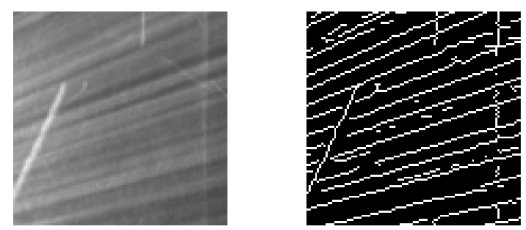

Figure 5: Left side: Detail of a fertilizer spreading image (extracted from the image presented in figure 3); Right side: Binary image obtained using the line detection filter.

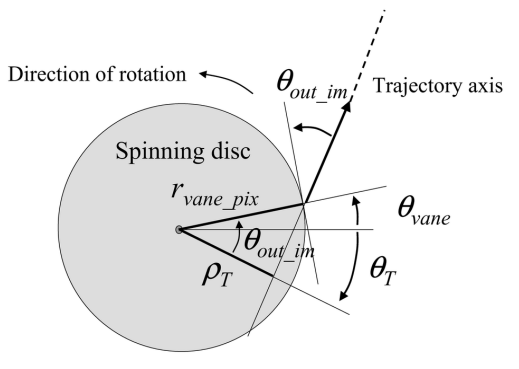

Figure 6: Geometrical relationship between the apparent outlet angle $\theta_{\text {out_im }}$ and the radius parameter $\rho_{T}$ of a trajectory.
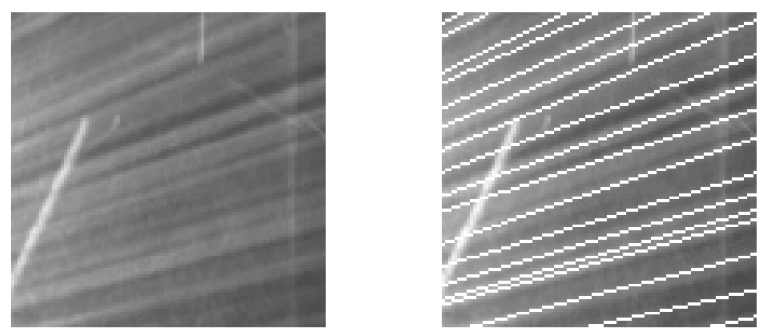

Figure 7: Detail of an image (left side) and its superposition with the identified trajectories (right side).

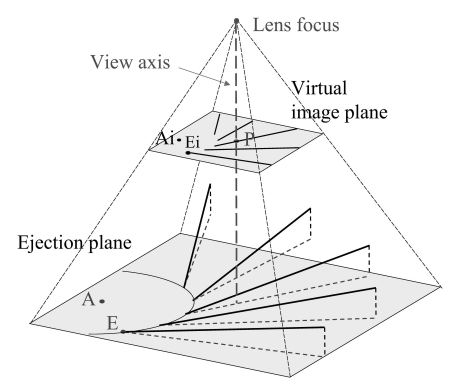

Figure 8: 3D simulation of tilted trajectories with orthogonal projections on a horizontal reference plane and with pinhole camera projections in the image plane; A, intersection point between the disc axle and the ejection plane; $\mathrm{E}$, point where a particle leaves the vane; $\mathrm{Ai}$, image of $\mathrm{A}$ in the CCD plane; Ei, image of $\mathrm{E}$ in the CCD plane; $\mathrm{P}$, principal point. 


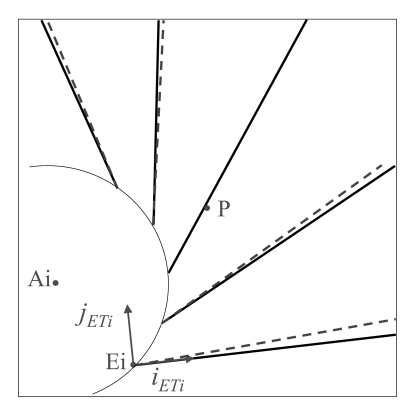

Figure 9: Pinhole camera projections of the trajectories (continuous lines) and of their horizontal components (dotted lines) corresponding to the situation represented in Fig. 7: Ai, image of $\mathrm{A}$ in the CCD plane, $\mathrm{P}$, principal point, $\left(\mathrm{Ei}, \boldsymbol{i}_{\boldsymbol{E} T \boldsymbol{i}}, \boldsymbol{j}_{\boldsymbol{E} T \boldsymbol{i}}\right)$ trajectory image frame.

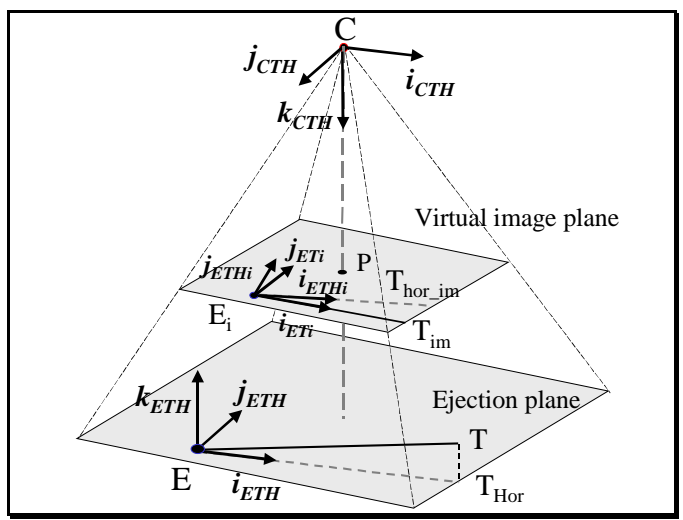

Figure 10:

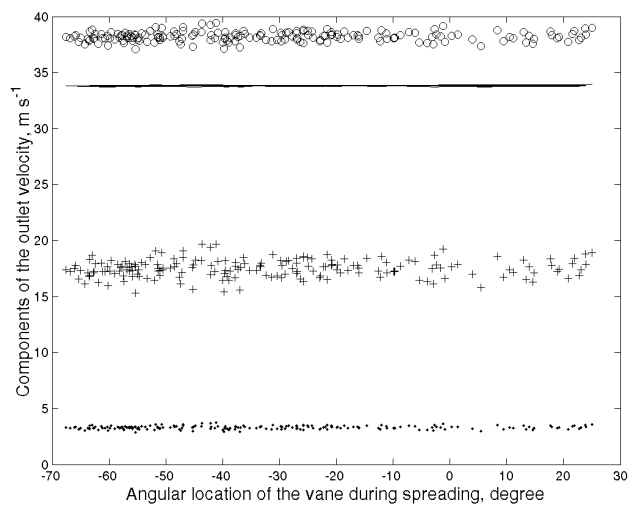

Figure 11: Outlet velocity and its components with respect to the angular location of the vane. Total velocity (o); tangential (-), radial (+) and vertical (·) components.
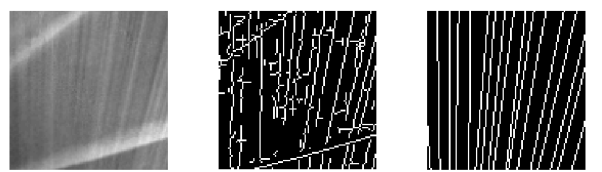

Figure 12: Example of results obtained when a canvas tarpaulin is used for the background. Left side: grey level image (detail); middle: binary image (detail); right side: identified trajectories. 

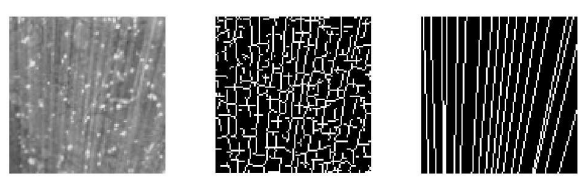

Figure 13: Example of results obtained when a light land scattered with white particles is used for the background. Left side: grey level image (detail); middle: binary image (detail); right side : identified trajectories. 